\title{
Conversion of Alcohols to Phosphorothiolates Using a Thioiminium Salt as Coupling Agent
}

\author{
Helen Grounds, Kristaps Ermanis, Sophie A. Newgas, and Michael J. Porter*i) \\ Department of Chemistry, University College London, Christopher Ingold Building, 20 Gordon Street, London WC1H 0AJ, U.K. \\ Supporting Information
}

ABSTRACT: We report a method for the direct and rapid conversion of primary and secondary alcohols to the corresponding phosphorothiolates in yields ranging from $64 \%$ to $97 \%$, using as a coupling agent the iminium salt prepared from $N, N$-dimethylthioformamide and Meerwein's salt. Selective reaction of primary alcohols in the presence of secondary alcohols is possible. The reaction of secondary alcohols proceeds stereospecifically with inversion

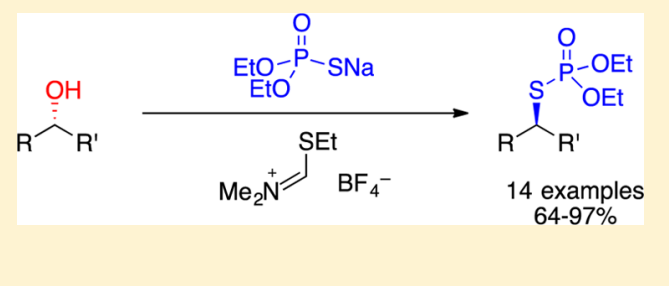
of configuration.

$\mathrm{T}$ he phosphorothiolate linkage, $\mathrm{RSPO}\left(\mathrm{OR}^{\prime}\right)_{2}$, has found extensive use in the synthesis of nucleic acid analogues, ${ }^{1,2}$ but until recently only a few examples of phosphorothiolates as synthetic intermediates were known. However, recent reports from the group of $\mathrm{Wu}$ have shown the utility of phosphorothioic acids as surrogates for $\mathrm{H}_{2} \mathrm{~S}$ in the synthesis of acylic and cyclic thioethers. ${ }^{3,4}$ This group has also shown that the $-\mathrm{SPO}(\mathrm{OR})_{2}$ moiety of allylic phosphorothiolates can be displaced by Grignard reagents ${ }^{5}$ or, in a palladium-catalyzed process, by fluoride. ${ }^{6}$

The most commonly used method for the synthesis of phosphorothiolate esters is the displacement of halide leaving groups by phosphorothiolate anions. Direct conversion of alcohols to phosphorothiolates under Mitsunobu conditions has also been reported. ${ }^{7,8} \mathrm{Wu}$ has reported a photochemical displacement of allylic hydroxy and methoxy groups by a phosphorothioic acid, ${ }^{5}$ and a gallium triflate catalyzed substitution of allylic, benzylic, and tertiary alcohols. ${ }^{9}$ More recently Han has reported a dehydrogenative palladium coupling of phosphonates with thiols. ${ }^{10}$

While the direct preparation of phosphorothiolates from alcohols without the need for prior activation is clearly advantageous, both the photochemical and gallium triflate methods suffer from both a lack of stereospecificity and a limited substrate scope (only allylic, benzylic, and tertiary alcohols react). Reported yields for the Mitsunobu reaction are only moderate $(40-77 \%)^{7}$

In this paper, we describe a simple procedure for the one-pot conversion of primary and secondary alcohols to phosphorothiolates, using a sulfanyliminium salt as an activating agent. Aliphatic alcohols as well as allylic and benzylic alcohols undergo the substitution reaction, and the reaction with secondary alcohols proceeds stereospecifically with inversion of configuration.

Our interest in the use of sulfanyliminium salts as activating agents for alcohols stemmed from a protocol we developed for the preparation of alkyl iodides from primary and secondary alcohols using salt $\mathbf{1}$ (Scheme 1). ${ }^{11}$ The presumed course of events in this iodination reaction involves attack of the alcohol
Scheme 1. Conversion of Alcohols to Alkyl Iodides

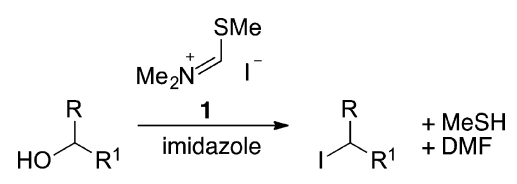

on the iminium ion, expulsion of methanethiol, and displacement of $\mathrm{N}, \mathrm{N}$-dimethylformamide by an iodide ion. Selective conversion of primary alcohols to iodides in the presence of secondary alcohols was possible. We also showed that, with secondary alcohols, the initial displacement occurred with inversion of configuration, although the enantiomeric purity of the product was rapidly eroded by subsequent $S_{N} 2$ reactions with further iodide ions.

Subsequently we modified this protocol by preparing a tetrafluoroborate analogue $\mathbf{2}$ of the iminium salt. Using this salt as an activating agent for a range of alcohols, we were able to use 1-phenyltetrazol-5-ylthiol as a nucleophile and to prepare the corresponding sulfides 3 directly. ${ }^{12}$ In this paper we describe the use of iminium salt $\mathbf{2}$ as an activating agent for the formation of phosphorothiolates from alcohols.

Our test substrate for the preparation of phosphorothiolates was 4-methylbenzyl alcohol $\mathbf{4 a}$, and the conditions employed were analogous to those in Scheme 2. Reaction of alcohol 4a

Scheme 2. Conversion of Alcohols to Tetrazolyl Sulfides

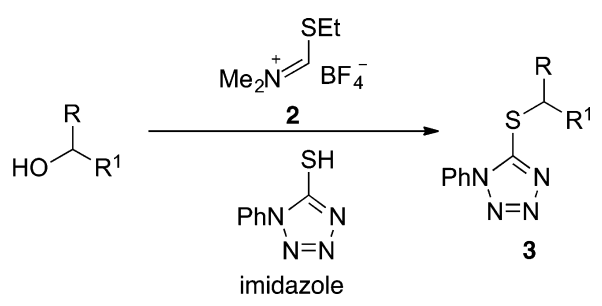

Received: July 3, 2017

Published: October 23, 2017 
Table 1. Formation of S-Alkyl O,O-Diethyl Phosphorothiolates

\begin{tabular}{|c|c|c|c|c|c|}
\hline & $\begin{array}{cl}\stackrel{O}{11} & 6(\mathrm{M}=\mathrm{H} \\
\text { EtO-P-PM } & \text { or } 7(\mathrm{M}= \\
\text { EtO' } & =\end{array}$ & = $\mathrm{Na}$ ) & $S^{-} \stackrel{\text { P }}{P}-$ OEt & & \\
\hline $4 a$ & $4-\mathrm{MeC}_{6} \mathrm{H}_{4}$ & $\mathrm{H}$ & A & $1 \mathrm{~h}$ & $94 \%$ \\
\hline $4 a$ & & & B & $0.5 \mathrm{~h}$ & $91 \%$ \\
\hline $4 b$ & $\mathrm{Ph}\left(\mathrm{CH}_{2}\right)_{2}$ & $\mathrm{H}$ & A & $1 \mathrm{~h}$ & $84 \%$ \\
\hline $4 b$ & & & B & $0.5 \mathrm{~h}$ & $97 \%$ \\
\hline 4c & $i-\operatorname{Pr}$ & $\mathrm{H}$ & $\mathrm{B}$ & $0.5 \mathrm{~h}$ & $88 \%$ \\
\hline 4d & $\mathrm{THPOCH}_{2}$ & $\mathrm{H}$ & $\mathrm{B}$ & $0.5 \mathrm{~h}$ & $88 \%$ \\
\hline $4 e$ & $\operatorname{BocNH}\left(\mathrm{CH}_{2}\right)_{2}$ & $\mathrm{H}$ & B & $3 \mathrm{~h}$ & $65 \%$ \\
\hline$( \pm)-\mathbf{4 f}$ & $n-\mathrm{C}_{6} \mathrm{H}_{13}$ & $\mathrm{Me}$ & B & $0.5 \mathrm{~h}$ & $78 \%$ \\
\hline$( \pm)-4 g$ & $\mathrm{Ph}$ & $\mathrm{Me}$ & B & $0.5 \mathrm{~h}$ & $75 \%$ \\
\hline$( \pm)-4 h$ & $\mathrm{Ph}\left(\mathrm{CH}_{2}\right)_{2}$ & $\mathrm{Me}$ & $\mathrm{B}$ & $0.5 \mathrm{~h}$ & $91 \%$ \\
\hline $4 \mathbf{i}$ & $(E)-\mathrm{PhCH}=\mathrm{CH}$ & $\mathrm{H}$ & B & $0.5 \mathrm{~h}$ & $77 \%$ \\
\hline$( \pm)-\mathbf{4 j}$ & $\mathrm{Ph}$ & $\mathrm{CH}=\mathrm{CH}_{2}$ & B & $0.5 \mathrm{~h}$ & $64 \%{ }^{b}$ \\
\hline$( \pm)-4 k$ & $(E)-\mathrm{PhCH}=\mathrm{CH}$ & $\mathrm{Me}$ & $\mathrm{B}$ & $0.5 \mathrm{~h}$ & $75 \%$ \\
\hline$( \pm)-41$ & $\mathrm{BnOCH}_{2} \mathrm{CH}(\mathrm{OH}) \mathrm{CH}_{2}$ & $\mathrm{H}$ & B & $1 \mathrm{~h}$ & $64 \%^{c}$ \\
\hline$( \pm)-4 m$ & $\mathrm{PhCH}(\mathrm{OH})\left(\mathrm{CH}_{2}\right)_{2}$ & $\mathrm{H}$ & A & $1.5 \mathrm{~h}$ & $0 \%{ }^{d}$ \\
\hline $\mathrm{HO}^{-}$ & & & B & $6 \mathrm{~h}$ & $0 \%^{e}$ \\
\hline $4 n$ & & & & & \\
\hline
\end{tabular}

${ }^{a}$ Conditions A: (EtO) $)_{2} \mathrm{POSH}$ (6) (1.1 equiv), $\mathrm{Me}_{2} \mathrm{NCHSEt}^{+} \mathrm{BF}_{4}{ }^{-}$(2) (1.1 equiv), imidazole (1.1 equiv), toluene, $90{ }^{\circ} \mathrm{C}$; Conditions $\mathrm{B}$ : $(\mathrm{EtO})_{2} \mathrm{POSNa}$ (7) (1.1 equiv), $\mathrm{Me}_{2} \mathrm{NCHSEt}^{+} \mathrm{BF}_{4}{ }^{-}$(2) (1.1 equiv), toluene, $90^{\circ} \mathrm{C}^{b}$ Total yield of a $1: 4$ mixture of secondary phosphorothiolate $5 \mathbf{j}$ and the primary phosphorothiolate $\mathbf{5 i}$ formed by an $\mathrm{S}_{\mathrm{N}} 2^{\prime}$ reaction. ${ }^{c}$ Using $\mathbf{2}$ (1.0 equiv) and 7 (1.0 equiv). ${ }^{d}$ 2-Phenyltetrahydrofuran was formed in $72 \%$ yield. ${ }^{e} \mathrm{EtSPO}(\mathrm{OEt})_{2}(9)$ formed in the reaction.

with diethyl phosphorothioic acid 6 (1.1 equiv), iminium salt 2 ( 1.1 equiv), and imidazole ( 1.1 equiv) in toluene at $90{ }^{\circ} \mathrm{C}$ (conditions A, Table 1) led, after $1 \mathrm{~h}$, to a $94 \%$ yield of the desired phosphorothiolate 5a. When 3-phenylpropan-1-ol $\mathbf{4 b}$ was subjected to the same conditions, an $84 \%$ yield of $\mathbf{5 b}$ was obtained.

For both of these reactions, it was found that two liquid phases formed and vigorous stirring was required in order for the reaction to proceed. However, when the acid $\mathbf{6}$ was replaced by its sodium salt 7 , the reaction proceeded in the absence of imidazole and without the formation of a second liquid phase. Under these conditions (conditions B) reactions were generally complete in $30 \mathrm{~min}$, with $\mathbf{5 a}$ and $\mathbf{5 b}$ formed in $91 \%$ yield and
97\% yield, respectively. These conditions were used to further explore the substrate scope of the reaction.

Three other primary alcohols, $\mathbf{4 c}$, $4 \mathrm{~d}$, and $\mathbf{4 e}$, were converted smoothly to the corresponding phosphorothiolates; acidsensitive functionalities in the form of a THP ether and a tert-butyl carbamate were unaffected. However, the reaction of the Boc-protected compound $\mathbf{4 e}$ was markedly slower than that of other substrates. Secondary alcohols $\mathbf{4 f}, \mathbf{4 g}$ and $\mathbf{4 h}$ also underwent the reaction to the corresponding phosphorothiolates. (E)-Cinnamyl alcohol $4 \mathbf{i}$ could be converted to the corresponding phosphorothiolate $\mathbf{5 i}$ in $77 \%$ yield. However, when 1-phenylprop-2-en-1-ol $\mathbf{4 j}$ was subjected to the reaction conditions, the major product was the same phosphorothiolate 5i resulting from allylic rearrangement. Secondary allylic 
alcohol 4k gave the expected phosphorothiolate $5 \mathbf{k}$ without allylic rearrangement in $75 \%$ yield.

The reaction was found to be selective for a primary alcohol in the presence of a secondary alcohol (41) when 1 equiv of 2 and 7 were used. However, when 1-phenyl-butane-1,4-diol (4m) was used tetrahydrofuran 8 was isolated as the major product $(72 \%)$ (Scheme 3$)$. In this case, it is presumed that

Scheme 3. Formation of 2-Phenyltetrahydrofuran ${ }^{a}$<smiles>OCCCC(O)c1ccccc1</smiles><smiles></smiles>

${ }^{a}$ Conditions: (EtO) ${ }_{2} \mathrm{POSH}$ (6) (1 equiv), $\mathrm{Me}_{2} \mathrm{NCHSEt}^{+} \mathrm{BF}_{4}{ }^{-}$(2) (1 equiv), imidazole ( 1 equiv), toluene, $90{ }^{\circ} \mathrm{C}, 72 \%$.

cyclization of the secondary alcohol to the activated primary position is more rapid than the intermolecular reaction with the sulfur nucleophile. Indeed, when the reaction of $4 \mathrm{~m}$ with 2 was carried out in the absence of sulfur nucleophile $\mathbf{6}$, the same tetrahydrofuran product 8 was obtained.

1,2:3,4-Di-O-isopropylidene-D-galactopyranose $4 n$ proved unreactive under the reaction conditions and was recovered unchanged. The low reactivity of leaving groups at the 6position of galactopyranose derivatives has been noted previously, ${ }^{13}$ although it should be noted that $4 \mathbf{n}$ has been shown to react with several sulfur nucleophiles under Mitsunobu conditions ${ }^{14}$ or with $\mathrm{PhSSPh} / \mathrm{Bu}_{3} \mathrm{P} .{ }^{15}$ In the present case, slow formation of $\mathrm{EtSPO}(\mathrm{OEt})_{2}$ (9) was observed, presumably from direct reaction of thioiminium salt 2 with phosphorothiolate anion 7 .

The stereochemical course of the reaction was investigated through reaction of $(S)$-1-phenylethanol $((S)-\mathbf{4 g}$, >99:1 er) with sodium salt 7 and iminium salt $\mathbf{2}$ (Scheme 4 ). The product

Scheme 4. Stereochemical Course of Reaction ${ }^{a}$

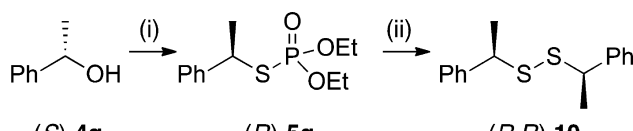

(S)-49

$(R)-5 \mathrm{~g}$

$(R, R)-10$

${ }^{a}(\mathrm{i})(\mathrm{EtO})_{2} \mathrm{POSNa}$ (7), (1.1 equiv), $\mathrm{Me}_{2} \mathrm{NCHSEt}^{+} \mathrm{BF}_{4}^{-}$(2) (1.1 equiv), toluene, $90{ }^{\circ} \mathrm{C}$, $83 \%$; (ii) $\mathrm{KOH}$ (4 equiv), THF, $\mathrm{H}_{2} \mathrm{O}, 72 \%$.

5g was obtained with 97:3 er. Upon hydrolysis of the sulfurphosphorus bond, 9 spontaneous oxidation occurred to give the known ${ }^{16}$ disulfide $(R, R)-10$, proving that the displacement had proceeded with inversion of configuration.

A similar selectivity was found when $(R)$-octan-2-ol $((R)-4 \mathbf{f}$, $>$ 98:2 er) was used: the product $\mathbf{5 f}$ was obtained with 97:3 er. However, when enantiopure $(R)-4 \mathbf{k}$ (prepared by enzymic resolution, ${ }^{17} 98.5: 1.5$ er) was subjected to the reaction conditions erosion of enantiopurity was seen with the desired phosphorothiolate isolated with 61:39 er.

The proposed mechanistic course of phosphorothiolate formation is outlined in Scheme 5. Nucleophilic addition of alcohol $\mathbf{4}$ to iminium ion $\mathbf{2}$ leads to the formation of the adduct 11, which can lose ethanethiolate (or, following protonation, ethanethiol) to give 12. Nucleophilic displacement of the DMF leaving group by phosphorothiolate ion 7 then affords the desired product $\mathbf{5}$.

In summary, a simple one-step method has been developed for the conversion of a wide range of primary and secondary
Scheme 5. Proposed Mechanism of Phosphorothiolate Formation

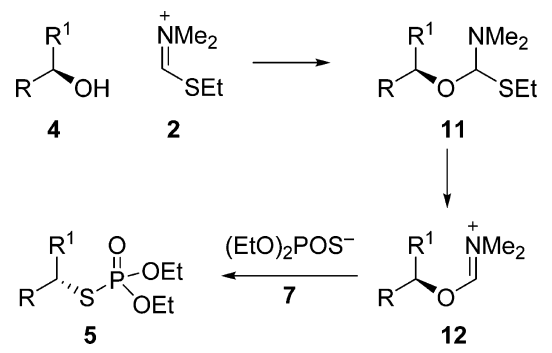

alcohols to the corresponding phosphorothiolates, with inversion of configuration. This methodology should facilitate the development of such compounds as synthetic intermediates.

\section{EXPERIMENTAL SECTION}

General Experimental Methods. All reactions were carried out under an inert atmosphere of $\mathrm{N}_{2}$ or Ar. All reagents were used as received. Thin layer chromatography was carried out using Merck silica-aluminum plates, with UV light $(254 \mathrm{~nm})$, and potassium permanganate or anisaldehyde for visualization. Column chromatography column chromatography was carried out using Merck Geduran Si 60 silica gel or Sigma-Aldrich weakly acidic activated alumina. Chiral stationary phase HPLC was performed using Chiralcel OD or Chiralpak AD-H $25 \mathrm{~cm}$ analytical columns. Infrared spectra were recorded using a Bruker Alpha ATR spectrometer. NMR spectra were recorded in $\mathrm{CDCl}_{3}$ using a Bruker AMX $300 \mathrm{MHz}$, Bruker AVANCE III $400 \mathrm{MHz}$, Bruker AVANCE $500 \mathrm{MHz}$, or Bruker AVANCE III 600 $\mathrm{MHz}$ spectrometer. Reference values for $\mathrm{CDCl}_{3}$ were taken as $\delta=7.27$ ppm for ${ }^{1} \mathrm{H}$ NMR spectroscopy and $\delta=77.2 \mathrm{ppm}$ for ${ }^{13} \mathrm{C}$ NMR spectroscopy. Coupling constants $(J)$ are given in $\mathrm{Hz}$. Where appropriate COSY and DEPT experiments were carried out to aid assignment. Mass spectrometry data were collected on a Waters Micromass LCT Premier XE time-of-flight instrument (ESI) or a Thermo Finnigan MAT900xp magnetic sector instrument (EI/CI). Elemental analysis was performed on an Exeter Analytical Inc. EA440 horizontal load analyzer. Optical rotations were obtained using a PerkinElmer 343 digital polarimeter and are reported in units of $10^{-1}$ $\operatorname{deg} \mathrm{cm}^{2} \mathrm{~g}^{-1}$.

$\mathrm{N}$-(Ethylsulfanylmethylene)- $\mathrm{N}, \mathrm{N}$-dimethylammonium tetrafluoroborate (2), ${ }^{12} \mathrm{O}, \mathrm{O}$-diethyl phosphorothioic acid (6), ${ }^{5}$ sodium $\mathrm{O}, \mathrm{O}$ diethyl phosphorothioate (7), ${ }^{4}$ 1-phenylprop-2-en-1-ol (4h), ${ }^{18}(R)-4$ phenylbut-3-en-2-ol $((R)-\mathbf{4 k}),{ }^{17}$ 4-benzyloxybutane-1,3-diol (4l) ${ }^{19}$ and 4-hydroxy-1-phenylbutan-1-one $(\mathbf{4 m})^{20}$ were synthesized according to reported literature procedures.

$(R)$-Octan-2-ol ((R)-4f) was purchased from Acros Organics, and its enantiomeric ratio was determined as $>98: 2$ by Mosher's ester analysis. $^{21}(S)$-1-Phenylethanol $((S)-\mathbf{4 g})$ was purchased from Acros Organics, and its enantiomeric ratio was determined as $>99: 1$ by chiral stationary phase HPLC (Chiralcel OD, 90:10 hexane/ ${ }^{i} \mathrm{PrOH}$; flow rate

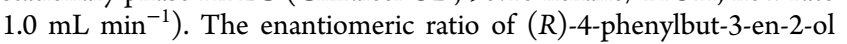
$((R)-4 \mathrm{k})$ was determined as $98.5: 1.5$ by chiral stationary phase HPLC

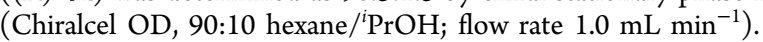

General Procedure for the Synthesis of Phosphorothiolates. A solution of alcohol $4(1.00 \mathrm{mmol})$ in toluene $(2 \mathrm{~mL})$ was treated with $(\mathrm{EtO})_{2} \mathrm{POSNa}(7)(211 \mathrm{mg}, 1.10 \mathrm{mmol})$ followed by iminium salt $2(226 \mathrm{mg}, 1.10 \mathrm{mmol})$ and heated using a silicone oil bath to 90 ${ }^{\circ} \mathrm{C}$ for $30 \mathrm{~min}$ (except where stated otherwise). The reaction was cooled to room temperature and concentrated in vacuo. Purification of the desired phosphorothiolates was achieved by flash column chromatography over $\mathrm{SiO}_{2}$ (or $\mathrm{Al}_{2} \mathrm{O}_{3}$ where stated).

O,O-Diethyl S-(4-Methylbenzyl) Phosphorothioate (5a). Eluent 30:70 EtOAc/hexanes; 5a (249 $\mathrm{mg}, 91 \%)$ obtained as a colorless oil; $R_{f}=0.30$ (20:80 EtOAc/hexanes); $\nu_{\max } / \mathrm{cm}^{-1} 2982,1514$, $1254,1012,969 ;{ }^{1} \mathrm{H} \mathrm{NMR}\left(600 \mathrm{MHz}, \mathrm{CDCl}_{3}\right) \delta 7.25(2 \mathrm{H}, \mathrm{d}, J=7.7)$, 
$7.13(2 \mathrm{H}, \mathrm{d}, J=7.7), 4.17-4.01(4 \mathrm{H}, \mathrm{m}), 4.01(2 \mathrm{H}, \mathrm{d}, J=13.6), 2.34$ $(3 \mathrm{H}, \mathrm{s}), 1.35(6 \mathrm{H}, \mathrm{t}, J=7.0) ;{ }^{13} \mathrm{C}\left\{{ }^{1} \mathrm{H}\right\}$ NMR $\left(150 \mathrm{MHz}^{\mathrm{CDCl}} \mathrm{CDCl}_{3}\right) \delta$ $137.5(\mathrm{C}), 134.4(\mathrm{~d}, J=5.0, \mathrm{C}), 129.4(\mathrm{CH}), 128.9(\mathrm{CH}), 63.6(\mathrm{~d}, J=$ 5.0, $\left.\mathrm{CH}_{2}\right), 34.9$ (d, $\left.J=2.5, \mathrm{CH}_{2}\right), 20.9\left(\mathrm{CH}_{3}\right), 16.1\left(\mathrm{~d}, J=6.3, \mathrm{CH}_{3}\right)$; ${ }^{31} \mathrm{P}$ NMR $\left(121 \mathrm{MHz}, \mathrm{CDCl}_{3}\right) \delta 26.9 ; \mathrm{m} / z$ (ESI+) $276(10 \%), 275$ $\left([\mathrm{M}+\mathrm{H}]^{+}, 100\right)$; HRMS (ESI $) m / z:[\mathrm{M}+\mathrm{H}]^{+}$calcd for $\mathrm{C}_{12} \mathrm{H}_{20} \mathrm{O}_{3}$ PS 275.0879; found 275.0871. Anal. Calcd for $\mathrm{C}_{12} \mathrm{H}_{19} \mathrm{O}_{3}$ PS: C, 52.54; H, 6.98. Found: C, 52.48; H, 7.11.

O,O-Diethyl S-(3-Phenylpropyl) Phosphorothioate (5b). Eluent 30:70 EtOAc/hexanes; 5b (280 mg, 97\%) obtained as a colorless oil; $R_{f}=0.34$ (40:60 EtOAc/hexanes); $\nu_{\max } / \mathrm{cm}^{-1} 2981,1453$, 1252, 1013, 967; ${ }^{1} \mathrm{H} \mathrm{NMR}\left(600 \mathrm{MHz}, \mathrm{CDCl}_{3}\right) \delta 7.31-7.28(2 \mathrm{H}, \mathrm{m})$, $7.22-7.18(3 \mathrm{H}, \mathrm{m}), 4.23-4.10(4 \mathrm{H}, \mathrm{m}), 2.89-2.81(2 \mathrm{H}, \mathrm{m}), 2.74$ $(2 \mathrm{H}, \mathrm{t}, J=7.4), 2.03(2 \mathrm{H}$, app. quintet, $J=7.5), 1.35(6 \mathrm{H}, \mathrm{t}, J=7.1)$; ${ }^{13} \mathrm{C}\left\{{ }^{1} \mathrm{H}\right\}$ NMR $\left(150 \mathrm{MHz}, \mathrm{CDCl}_{3}\right) \delta 140.9(\mathrm{C}), 128.6(\mathrm{CH}), 128.6$ $(\mathrm{CH}), 126.2(\mathrm{CH}), 63.6\left(\mathrm{~d}, J=5.0, \mathrm{CH}_{2}\right), 34.6\left(\mathrm{CH}_{2}\right), 32.3(\mathrm{~d}, J=$ 3.8, $\left.\mathrm{CH}_{2}\right), 30.3\left(\mathrm{~d}, J=2.5, \mathrm{CH}_{2}\right), 16.2\left(\mathrm{~d}, J=6.3, \mathrm{CH}_{3}\right) ;{ }^{31} \mathrm{P}$ NMR $\left(121 \mathrm{MHz}, \mathrm{CDCl}_{3}\right) \delta 29.0 ; \mathrm{m} / z(\mathrm{EI}) 288\left(\mathrm{M}^{+}, 47 \%\right), 118([\mathrm{M}-$ $\left.\left.(\mathrm{EtO})_{2} \mathrm{POSH}\right]^{+}, 100\right), 91\left(\mathrm{PhCH}_{2}^{+}, 26\right)$; HRMS (EI) $\mathrm{m} / z:\left[\mathrm{M}^{+}\right]$calcd for $\mathrm{C}_{13} \mathrm{H}_{21} \mathrm{O}_{3} \mathrm{PS}$ 288.0944; found 288.0948. Anal. Calcd for $\mathrm{C}_{13} \mathrm{H}_{21} \mathrm{O}_{3}$ PS: C, 54.15; H, 7.34. Found: C, 54.36; H, 7.47.

O,O-Diethyl S-(2-Methylpropyl) Phosphorothioate (5c). Eluent 30:70 EtOAc/hexanes; 5c (198 mg, 88\%) obtained as a colorless oil; $R_{f}=0.29$ (20:80 EtOAc/hexanes); $\nu_{\max } / \mathrm{cm}^{-1} 2960,1388$, 1258, 1163, 1014, 967; ${ }^{1} \mathrm{H}$ NMR (300 MHz, $\left.\mathrm{CDCl}_{3}\right) \delta 4.23-4.07$ $(4 \mathrm{H}, \mathrm{m}), 2.73(2 \mathrm{H}, \mathrm{dd}, J=13.1,6.8), 1.90(1 \mathrm{H}$, app. nonet, $J=6.7)$, $1.36(6 \mathrm{H}, \mathrm{t}, J=7.1), 1.01(6 \mathrm{H}, \mathrm{d}, J=6.6) ;{ }^{13} \mathrm{C}\left\{{ }^{1} \mathrm{H}\right\} \mathrm{NMR}(125 \mathrm{MHz}$, $\left.\mathrm{CDCl}_{3}\right) \delta 63.5\left(\mathrm{~d}, J=5.2, \mathrm{CH}_{2}\right), 39.5\left(\mathrm{~d}, J=3.8, \mathrm{CH}_{2}\right), 29.6(\mathrm{~d}, J=$ 6.0, $\mathrm{CH}), 21.7\left(\mathrm{CH}_{3}\right), 16.2\left(\mathrm{~d}, J=6.8, \mathrm{CH}_{3}\right) ;{ }^{31} \mathrm{P}$ NMR $(121 \mathrm{MHz}$, $\left.\mathrm{CDCl}_{3}\right) \delta 28.5 ; \mathrm{m} / z(\mathrm{CI}) 227\left([\mathrm{M}+\mathrm{H}]^{+}, 100 \%\right)$; HRMS (CI) $[\mathrm{M}+$ $\mathrm{H}]^{+}$calcd for $\mathrm{C}_{8} \mathrm{H}_{20} \mathrm{O}_{3} \mathrm{PS} 227.0865$; found 227.0865.

O,O-Diethyl S-(2-(Tetrahydro-2H-pyran-2-yloxy)ethyl) Phosphorothioate (5d). Eluent 25:75 EtOAc/hexanes; 5d (262 mg, 88\%) obtained as a colorless oil; $R_{f}=0.18(25: 75 \mathrm{EtOAc} /$ hexanes $) ; \nu_{\max } /$ $\mathrm{cm}^{-1} 2979,1455,1352,1252,1209,1127,1081,1014,967 ;{ }^{1} \mathrm{H}$ NMR $\left(300 \mathrm{MHz}, \mathrm{CDCl}_{3}\right) \delta 4.65(1 \mathrm{H}, \mathrm{t}, J=3.4), 4.29-4.08(4 \mathrm{H}, \mathrm{m}), 3.98-$ $3.82(2 \mathrm{H}, \mathrm{m}), 3.65(1 \mathrm{H}, \mathrm{dt}, J=10.4,6.5), 3.52(1 \mathrm{H}, \mathrm{m}), 3.00(2 \mathrm{H}, \mathrm{dt}$, $J=15.1,6.5), 1.83-1.60(6 \mathrm{H}, \mathrm{m}), 1.37(6 \mathrm{H}, \mathrm{t}, J=7.1) ;{ }^{13} \mathrm{C}\left\{{ }^{1} \mathrm{H}\right\} \mathrm{NMR}$ $\left(75 \mathrm{MHz}, \mathrm{CDCl}_{3}\right) \delta 98.9(\mathrm{CH}), 66.8\left(\mathrm{~d}, J=5.0, \mathrm{CH}_{2}\right), 63.7(\mathrm{~d}, J=$ 5.9, $\left.\mathrm{CH}_{2}\right), 62.3\left(\mathrm{CH}_{2}\right), 30.7\left(\mathrm{~d}, J=3.9, \mathrm{CH}_{2}\right), 30.6\left(\mathrm{CH}_{2}\right), 25.5$ $\left(\mathrm{CH}_{2}\right), 19.4\left(\mathrm{CH}_{2}\right), 16.1\left(\mathrm{~d}, J=6.8, \mathrm{CH}_{3}\right) ;{ }^{31} \mathrm{P}$ NMR $(121 \mathrm{MHz}$, $\left.\mathrm{CDCl}_{3}\right) \delta 27.9 ; \mathrm{m} / z(\mathrm{ES}+) 299\left([\mathrm{M}+\mathrm{H}]^{+}, 100 \%\right) ; \mathrm{HRMS}(\mathrm{ES}+)[\mathrm{M}$ $+\mathrm{H}]^{+}$calcd for $\mathrm{C}_{11} \mathrm{H}_{24} \mathrm{O}_{5}$ PS 299.1082; found 299.1089. Anal. Calcd for $\mathrm{C}_{11} \mathrm{H}_{23} \mathrm{O}_{5} \mathrm{PS}$ : C, 44.28; $\mathrm{H}, 7.77$. Found: $\mathrm{C}, 44.21$; $\mathrm{H}, 7.94$.

tert-Butyl (3-((Diethoxyphosphoryl)thio)propyl)carbamate (5e). Reaction conducted at $90{ }^{\circ} \mathrm{C}$ for $180 \mathrm{~min}$. Purified by flash column chromatography on alumina (30:70 EtOAc/hexanes) to give O,O,S-triethyl phosphorothioate (28 mg, 14\%) and 5e (214 mg, 65\%) as a colorless oil; $R_{f}=0.33$ (50:50 EtOAc/hexanes); $\nu_{\max } / \mathrm{cm}^{-1} 3327$, 2978, 2933, 1708, 1692, 1519, 1478, 1444, 1391, 1365, 1245, 1164, 1097,$967 ;{ }^{1} \mathrm{H}$ NMR $\left(600 \mathrm{MHz}, \mathrm{CDCl}_{3}\right) \delta 4.94(1 \mathrm{H}, \mathrm{br} \mathrm{s}), 4.24-4.12$ $(4 \mathrm{H}, \mathrm{m}), 3.27-3.22(2 \mathrm{H}, \mathrm{m}), 2.87(2 \mathrm{H}, \mathrm{dt}, J=15.7,7.0), 1.88(2 \mathrm{H}$, quintet, $J=6.7), 1.43(9 \mathrm{H}, \mathrm{s}), 1.36(6 \mathrm{H}, \mathrm{t}, J=7.1) ;{ }^{13} \mathrm{C}\left\{{ }^{1} \mathrm{H}\right\} \mathrm{NMR}$ $\left(150 \mathrm{MHz}, \mathrm{CDCl}_{3}\right) \delta 156.1(\mathrm{C}), 79.4(\mathrm{C}), 63.8\left(\mathrm{~d}, J=6.2, \mathrm{CH}_{2}\right), 38.7$ $\left(\mathrm{CH}_{2}\right), 31.2\left(\mathrm{CH}_{2}\right), 28.5\left(\mathrm{CH}_{3}\right), 28.2\left(\mathrm{CH}_{2}\right), 16.2\left(\mathrm{~d}, J=7.2, \mathrm{CH}_{3}\right)$; ${ }^{31} \mathrm{P}$ NMR $\left(121 \mathrm{MHz}, \mathrm{CDCl}_{3}\right) \delta 28.1 ; \mathrm{m} / z(\mathrm{ESI}) 328\left([\mathrm{M}+\mathrm{H}]^{+}\right.$, $100 \%)$; HRMS (ESI) $[\mathrm{M}+\mathrm{H}]^{+}$calcd for $\mathrm{C}_{12} \mathrm{H}_{27} \mathrm{NO}_{5} \mathrm{PS} 328.1348$; found 328.1351. Anal. Calcd for $\mathrm{C}_{12} \mathrm{H}_{26} \mathrm{NO}_{5} \mathrm{PS}$ : C, 44.03; H, 8.00; N, 4.28. Found: C, 44.30; H, 8.20; N, 4.49.

O,O-Diethyl S-Octan-2-yl Phosphorothioate (5f). ${ }^{22}$ Eluent 0:100 to 20:80 EtOAc/hexanes; ( \pm )-5f $(220 \mathrm{mg}, 78 \%)$ obtained as a colorless oil. The NMR data agree with those reported in the literature. ${ }^{22}$

Reaction repeated using $(R)$-octan-2-ol on a $0.82 \mathrm{mmol}$ scale. Eluent 0:100 to 20:80 EtOAc/hexanes; $(S)-5 f(130 \mathrm{mg}, 72 \%)$ obtained as a colorless oil. $[\alpha]_{\mathrm{D}}^{20}+7\left(c 1.03,{ }^{n} \mathrm{Bu}_{2} \mathrm{O}\right)\left[\right.$ lit. ${ }^{22}+6.59$ (no conc. reported, ${ }^{n} \mathrm{Bu}_{2} \mathrm{O}$ )]; HPLC analysis (Chiralpak AD-H; 90:10

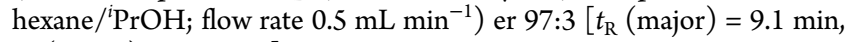
$t_{\mathrm{R}}($ minor $\left.)=8.6 \mathrm{~min}\right]$.
O,O-Diethyl S-(1-Phenylethyl) Phosphorothioate (5g). ${ }^{23}$ Eluent 30:70 EtOAc/hexanes; $( \pm)-5 g$ (206 $\mathrm{mg}, 75 \%)$ obtained as a colorless oil. The NMR data agree with those reported in the literature. $^{23}$

Reaction repeated using (S)-1-phenylethanol. Eluent 30:70 EtOAc/ hexanes; $(R)-5 \mathrm{~g}(226 \mathrm{mg}, 83 \%)$ obtained as a colorless oil. $[\alpha]_{\mathrm{D}}^{20}$ +160.4 (c 1.03, $\mathrm{CH}_{2} \mathrm{Cl}_{2}$ ); HPLC analysis (Chiralcel OD; 99:1

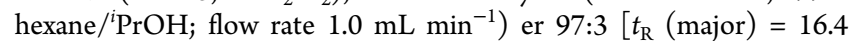
$\min , t_{\mathrm{R}}($ minor $\left.)=22.2 \mathrm{~min}\right]$.

( \pm )-O,O-Diethyl S-(4-Phenylbutan-2-yl) Phosphorothioate (5h). Eluent 30:70 EtOAc/hexanes; 5h (275 mg, 91\%) obtained as a colorless oil; $R_{f}=0.41$ (40:60 EtOAc/hexanes); $\nu_{\max } / \mathrm{cm}^{-1} 2981$, 1453, 1252, 1016, 966; ${ }^{1} \mathrm{H}$ NMR $\left(600 \mathrm{MHz}, \mathrm{CDCl}_{3}\right) \delta 7.30-7.27$ $(2 \mathrm{H}, \mathrm{m}), 7.21-7.19(3 \mathrm{H}, \mathrm{m}), 4.23-4.09(4 \mathrm{H}, \mathrm{m}), 3.37$ (1H, app d.sextet, $J=13.3,6.7), 2.83-2.70(2 \mathrm{H}, \mathrm{m}), 2.07-1.90(2 \mathrm{H}, \mathrm{m}), 1.49$ $(3 \mathrm{H}, \mathrm{d}, J=6.8), 1.36(3 \mathrm{H}, \mathrm{t}, J=7.1), 1.34(3 \mathrm{H}, \mathrm{t}, J=7.1) ;{ }^{13} \mathrm{C}\left\{{ }^{1} \mathrm{H}\right\}$ NMR $\left(150 \mathrm{MHz}, \mathrm{CDCl}_{3}\right) \delta 141.4(\mathrm{C}), 128.6(\mathrm{CH}), 128.5(\mathrm{CH})$, $126.2(\mathrm{CH}), 63.60\left(\mathrm{~d}, J=5.0, \mathrm{CH}_{2}\right), 63.58\left(\mathrm{~d}, J=5.0, \mathrm{CH}_{2}\right), 42.6(\mathrm{~d}, J$ $=2.5, \mathrm{CH}), 40.2\left(\mathrm{~d}, J=7.5, \mathrm{CH}_{2}\right), 33.3\left(\mathrm{CH}_{2}\right), 23.7\left(\mathrm{~d}, J=3.8, \mathrm{CH}_{3}\right)$, $16.20\left(\mathrm{~d}, J=5.0, \mathrm{CH}_{3}\right), 16.17\left(\mathrm{~d}, J=5.0, \mathrm{CH}_{3}\right) ;{ }^{31} \mathrm{P}$ NMR $(121 \mathrm{MHz}$, $\left.\mathrm{CDCl}_{3}\right) \delta 27.3 ; \mathrm{m} / z(\mathrm{EI}) 302\left(\mathrm{M}^{+}, 17 \%\right), 132\left(\left[\mathrm{M}-(\mathrm{EtO})_{2} \mathrm{POSH}\right]^{+}\right.$, 100), $117\left(\mathrm{PhCH}_{2} \mathrm{CHCH}^{+}, 68\right), 91\left(\mathrm{PhCH}_{2}{ }^{+}, 34\right)$; HRMS (EI) $[\mathrm{M}]^{+}$ calcd for $\mathrm{C}_{14} \mathrm{H}_{23} \mathrm{O}_{3} \mathrm{PS}$ 302.1106; found 302.1106. Anal. Calcd for $\mathrm{C}_{14} \mathrm{H}_{23} \mathrm{O}_{3} \mathrm{PS}$ : C, 55.61; $\mathrm{H}, 7.67$. Found $\mathrm{C}, 55.71 ; \mathrm{H}, 7.80$.

(E)-O,O-Diethyl S-(3-Phenylprop-2-enyl) Phosphorothioate (5i). ${ }^{23}$ Eluent 30:70 EtOAc/hexanes; $5 \mathbf{i}(219 \mathrm{mg}, 77 \%)$ obtained as a pale yellow oil. The NMR data agree with those reported in the literature. ${ }^{23}$

(士)-O,O-Diethyl S-(1-Phenylprop-2-enyl) Phosphorothioate (5j). Eluent 30:70 EtOAc/hexanes; obtained $5 \mathbf{i}(154 \mathrm{mg}, 51 \%)$, and $5 \mathbf{j}$ (39 mg, 13\%) as a colorless oil; $R_{f}=0.42$ (40:60 EtOAc/hexanes); $\nu_{\max } / \mathrm{cm}^{-1} 2979,1450,1389,1249,1160,1096,1008,966 ;{ }^{1} \mathrm{H}$ NMR $\left(300 \mathrm{MHz}, \mathrm{CDCl}_{3}\right) \delta 7.37-7.30(5 \mathrm{H}, \mathrm{m}), 6.18(1 \mathrm{H}, \mathrm{ddd}, J=16.9$, $10.1,7.4), 5.26(1 \mathrm{H}, \mathrm{dt}, J=16.9,1.2), 5.20(1 \mathrm{H}, \mathrm{dt}, J=10.1,1.0), 4.99$ $(1 \mathrm{H}, \mathrm{ddt}, J=11.4,7.4,1.2), 4.11-3.95(4 \mathrm{H}, \mathrm{m}), 1.263(3 \mathrm{H}, \mathrm{td}, J=7.1$, 0.9), $1.258(3 \mathrm{H}, \mathrm{td}, J=7.1,0.9) ;{ }^{13} \mathrm{C}\left\{{ }^{1} \mathrm{H}\right\} \operatorname{NMR}\left(125 \mathrm{MHz} \mathrm{CDCl}_{3}\right) \delta$ $140.6(\mathrm{~d}, J=4.0, \mathrm{C}), 137.9(\mathrm{~d}, J=5.2, \mathrm{CH}), 128.8(\mathrm{CH}), 128.0(\mathrm{CH})$, $127.8(\mathrm{CH}), 117.0\left(\mathrm{CH}_{2}\right), 63.62\left(\mathrm{~d}, J 6.0, \mathrm{CH}_{2}\right), 63.58(\mathrm{~d}, J=5.8$, $\left.\mathrm{CH}_{2}\right), 53.1(\mathrm{~d}, J=3.3, \mathrm{CH}), 16.0\left(\mathrm{~d}, J=7.4, \mathrm{CH}_{3}\right) ;{ }^{31} \mathrm{P}$ NMR $(121$ $\left.\mathrm{MHz}, \mathrm{CDCl}_{3}\right) \quad \delta 25.3 ; \mathrm{m} / z$ (EI) $286\left(\mathrm{M}^{+}, 19 \%\right), 117$ ([M$\left.\left.(\mathrm{EtO})_{2} \mathrm{POS}\right]^{+}, 100\right)$; HRMS (EI) $[\mathrm{M}]^{+}$calcd for $\mathrm{C}_{13} \mathrm{H}_{19} \mathrm{O}_{3} \mathrm{PS}$ 286.0787; found 286.0787 .

(E)-O,O-Diethyl S-(4-Phenylbut-3-en-2-yl) Phosphorothioate (5k). ${ }^{5}$ Eluent 20:80 EtOAc/hexanes; ( \pm )-5k (225 mg, 75\%) obtained as a colorless oil. The NMR data agree with those reported in the literature. ${ }^{5}$

Reaction repeated using $(R)-4 \mathbf{k}$ (er 98.5:1.5) ${ }^{17}$ to give $5 \mathbf{k}(141 \mathrm{mg}$, $69 \%$ ) as a colorless oil. HPLC analysis (Chiralcel OD; 97:3

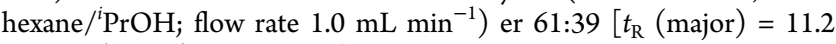
$\min , t_{\mathrm{R}}($ minor $\left.)=13.7 \mathrm{~min}\right]$.

O,O-Diethyl S-(4-Benzyloxy-3-hydroxybutyl) Phosphorothioate (5I). Reaction conducted at $90{ }^{\circ} \mathrm{C}$ for $60 \mathrm{~min}$ using 1.0 equiv of 2 and 1.0 equiv of 7 . Purified by flash column chromatography on alumina (30:70 EtOAc/hexanes) to give $51(222 \mathrm{mg}, 64 \%)$ as a colorless oil; $R_{f}=0.31$ (60:40 EtOAc/hexanes); $\nu_{\max } / \mathrm{cm}^{-1} 3397,2983$, 2905, 2862, 1453, 1237, 1162, 1010, 967; ${ }^{1} \mathrm{H}$ NMR $(600 \mathrm{MHz}$, $\left.\mathrm{CDCl}_{3}\right) \delta 7.38-7.28(5 \mathrm{H}, \mathrm{m}), 4.23-4.10(4 \mathrm{H}, \mathrm{m}), 3.99(1 \mathrm{H}, \mathrm{ddt}, J$ 8.8, 7.2, 3.7), $3.51(1 \mathrm{H}, \mathrm{dd}, J=9.5,3.4), 3.41(1 \mathrm{H}, \mathrm{dd}, J=9.5,7.0)$, 3.04-2.97 (2H, m), 1.89-1.78 (2H, m), $1.37-1.35(6 \mathrm{H}, \mathrm{m}) ;{ }^{13} \mathrm{C}\left\{{ }^{1} \mathrm{H}\right\}$ NMR $\left(150 \mathrm{MHz}, \mathrm{CDCl}_{3}\right) \delta 138.0(\mathrm{C}), 128.6(\mathrm{CH}), 128.0(\mathrm{CH})$, $127.9(\mathrm{CH}), 74.2\left(\mathrm{CH}_{2}\right), 73.5\left(\mathrm{CH}_{2}\right), 68.3(\mathrm{CH}), 63.9(\mathrm{~d}, J=6.1$, $\left.\mathrm{CH}_{2}\right), 63.8\left(\mathrm{~d}, J=6.2, \mathrm{CH}_{2}\right), 34.6\left(\mathrm{~d}, J=4.2, \mathrm{CH}_{2}\right), 27.2(\mathrm{~d}, J=3.9$, $\left.\mathrm{CH}_{2}\right), 16.22\left(\mathrm{~d}, J=4.7, \mathrm{CH}_{3}\right), 16.17\left(\mathrm{~d}, J=4.8, \mathrm{CH}_{3}\right) ;{ }^{31} \mathrm{P}$ NMR $(162$ $\left.\mathrm{MHz} \mathrm{CDCl}_{3}\right) \delta 28.5 ; \mathrm{m} / z(\mathrm{ES}+) 349\left([\mathrm{M}+\mathrm{H}]^{+}, 100 \%\right)$; HRMS (ES +) $[\mathrm{M}+\mathrm{H}]^{+}$calcd for $\mathrm{C}_{15} \mathrm{H}_{26} \mathrm{O}_{5} \mathrm{PS} 349.1239$; found 349.1241. Anal. Calcd for $\mathrm{C}_{15} \mathrm{H}_{25} \mathrm{O}_{5}$ PS: C, 51.71; H, 7.23. Found: C, 51.72; H, 7.31.

2-Phenyltetrahydrofuran (8). ${ }^{24}$ Purified by flash chromatography on alumina (5:95 EtOAc/hexanes) to give $8(83 \mathrm{mg}, 72 \%)$ as a colorless oil. The NMR data agree with those reported in the literature. $^{24}$ 
$(R, R)-(+)$-Di(1-phenylethyl) Disulfide (10). ${ }^{16}$ A solution of $(R)$ $5 \mathrm{~g}(50 \mathrm{mg}, 0.182 \mathrm{mmol})$ in THF $(0.66 \mathrm{~mL})$ was treated with a solution of $\mathrm{KOH}(41 \mathrm{mg}, 0.731 \mathrm{mmol})$ in $\mathrm{H}_{2} \mathrm{O}(0.34 \mathrm{~mL})$ and stirred at $\mathrm{rt}$ for $6 \mathrm{~h}$. After this time the solution was loaded directly onto a column and purified by flash column chromatography (0-10\% EtOAc/Hexanes, $\left.\mathrm{SiO}_{2}\right)$ to give $(R, R)-10(18 \mathrm{mg}, 72 \%)$ as a colorless oil. $[\alpha]_{\mathrm{D}}^{20}+229(c 0.65$, EtOH $)\left(\right.$ lit. $^{16}[\alpha]_{\mathrm{D}}^{20}+277(c 1.16$, EtOH $\left.)\right)$. The NMR data agree with those reported in the literature. ${ }^{16}$

\section{ASSOCIATED CONTENT}

\section{S Supporting Information}

The Supporting Information is available free of charge on the ACS Publications website at DOI: 10.1021/acs.joc.7b01657.

Copies of ${ }^{1} \mathrm{H}$ and ${ }^{13} \mathrm{C}\left\{{ }^{1} \mathrm{H}\right\}$ NMR spectra for all compounds synthesized (PDF)

\section{AUTHOR INFORMATION}

\section{Corresponding Author}

*E-mail: m.j.porter@ucl.ac.uk.

\section{ORCID $\odot$}

Michael J. Porter: 0000-0002-0376-5434

Notes

The authors declare no competing financial interest.

\section{ACKNOWLEDGMENTS}

We thank EPSRC (Grant Number EP/P000428/1) for funding, Ms. Naomi Gaynor for preliminary experiments, Dr. Abil Aliev for assistance with NMR spectroscopy, and Dr. Kersti Karu for mass spectrometry.

\section{REFERENCES}

(1) Li, N.-S.; Frederiksen, J. K.; Piccirilli, J. A. Acc. Chem. Res. 2011, 44, 1257.

(2) Gaynor, J. W.; Cosstick, R. Curr. Org. Chem. 2008, 12, 291.

(3) Robertson, F.; Wu, J. Org. Lett. 2010, 12, 2668.

(4) Robertson, F. J.; Wu, J. J. Am. Chem. Soc. 2012, 134, 2775.

(5) Han, X.; Zhang, Y.; Wu, J. J. Am. Chem. Soc. 2010, 132, 4104.

(6) Lauer, A. M.; Wu, J. Org. Lett. 2012, 14, 5138.

(7) Młotkowska, B.; Wartałowska-Graczyk. J. Prakt. Chem. (Leipzig) 1987, 329, 735 .

(8) Hayakawa, Y.; Hirabayashi, Y.; Hyodo, M.; Yamashita, S.; Matsunami, T.; Cui, D.-M.; Kawai, R.; Kodama, H. Eur. J. Org. Chem. 2006, 2006, 3834.

(9) Han, X.; Wu, J. Org. Lett. 2010, 12, 5780.

(10) Zhu, Y.; Chen, T.; Li, S.; Shimada, S.; Han, L.-B. J. Am. Chem. Soc. 2016, 138, 5825.

(11) Ellwood, A. R.; Porter, M. J. J. Org. Chem. 2009, 74, 7982.

(12) Ellwood, A. R; Porter, M. J. Org. Biomol. Chem. 2011, 9, 379.

(13) Richardson, A. C. Carbohydr. Res. 1969, 10, 395.

(14) (a) Rollin, P. Tetrahedron Lett. 1986, 27, 4169. (b) von Itzstein, M.; Jenkins, M. J.; Mocerino, M. Carbohydr. Res. 1990, 208, 287. (c) Gueyrard, D.; Tatibouët, A.; Gareau, Y.; Rollin, P. Org. Lett. 1999, 1,521 .

(15) González, F. S.; Baer, H. H. Carbohydr. Res. 1990, 202, 33.

(16) Harpp, D. N.; Smith, R. A. J. Am. Chem. Soc. 1982, 104, 6045.

(17) Onaran, M. B.; Seto, C. T. J. Org. Chem. 2003, 68, 8136.

(18) Jautze, S.; Peters, R. Angew. Chem., Int. Ed. 2008, 47, 9284.

(19) Giordano, M.; Iadonisi, A. J. Org. Chem. 2014, 79, 213.

(20) Hans, J.; Wallace, E. M.; Zhao, Q.; Lyssikatos, J. P.; Aicher, T. D.; Laird, E. R.; Robinson, J.; Allen, S. Mitotic Kinesin Inhibitors and Methods of Use Thereof. U.S. Patent 7,449,486, Nov 11, 2008.

(21) Dale, J. A.; Dull, D. L.; Mosher, H. S. J. Org. Chem. 1969, 34, 2543.

(22) LeGras, P. G.; Dyer, R. L.; Clifford, P. J.; Hall, C. D. J. Chem. Soc., Perkin Trans. 2 1973, 2, 2064.
(23) Yadav, V. K.; Balamurugan, R.; Parvez, M.; Yamdagni, R. J. Chem. Soc., Perkin Trans. 2001, 1, 323.

(24) Kundu, R.; Ball, Z. T. Org. Lett. 2010, 12, 2460. 